\title{
TEXTURAL AND MICROSTRUCTURAL EVOLUTION DURING COLD-ROLLING OF PURE NICKEL
}

\author{
N. Hansen ${ }^{1)}$, \\ D. Juul Jersen ${ }^{1)}$ and \\ D.A. Hughes ${ }^{2}$ \\ 1) Materials Department, Ris $\varnothing$ National Laboratory, DK-4000 Roskilde, Denmark \\ 2) Materials Department, Sandia National Laboratories, Livermore, CA 94550, U.S.A.

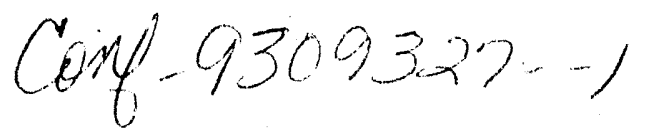 \\ Keywords: Nickel, cold-rolling, microstructure, texture, deformation pattern, geometrically \\ necessary boundaries, cell blocks.
}

\section{ABSTRACT}

High purity nickel (99.99\%) with a grain size about $100 \mu \mathrm{m}$ has been deformed by cold-rolling from $37 \%$ to $98 \%$ reductions. The deformation microstructures and the crystallographic texture have been characterised using transmission electron microscopy and neutron diffraction, respectively. The microstructural evolution has been described within a general framework which consists of a grain subdivision by dislocation boundaries on a finer and finer scale with increasing strain. The influence of this deformation pattern on the texture development is discussed.

\section{INTRODUCTION}

It is a general finding that predicted deformation textures differ from observed textures. Such discrepancies have generally been studied by developing various types of texture models which are variants of the classical models such as the Taylor-Bishop model and the self consistent model (1). In these modified models many factors have been taken into account such as the deformation geometry, changes in grain shape and grain-grain interaction. These approaches to reconcile texture predictions and observations have also included the microstructural evolution as a supplementary factor affecting the texture development (2). It is the main aim of the present paper to extend such microstructural considerations based on (i) a recently established framework for the microstructural evolution ( 3 - 6) and (ii) detailed observations by transmission electron microscopy of high purity nickel deformed by cold-rolling over a large strain range $(5,7)$. This discussion of microstructural affects will be preceeded by a section on texture observations.

\section{EXPERIMENTAL PROCEDURES}

High purity nickel $(99.99 \%)$ with a recrystallized grain size of about $100 \mu \mathrm{m}$ has been deformed by cold-rolling from $37 \%$ to $98 \%$ reductions (von Mises effective strain $0.5-4.5$ ). The deformation has been done using $75-\mathrm{mm}$-diameter rolls operating at $25 \mathrm{rpm}$ with an average of $15 \%$ cold reduction (c.r.) per pass. The crystallographic texture has been analyzed by neutron diffraction of 


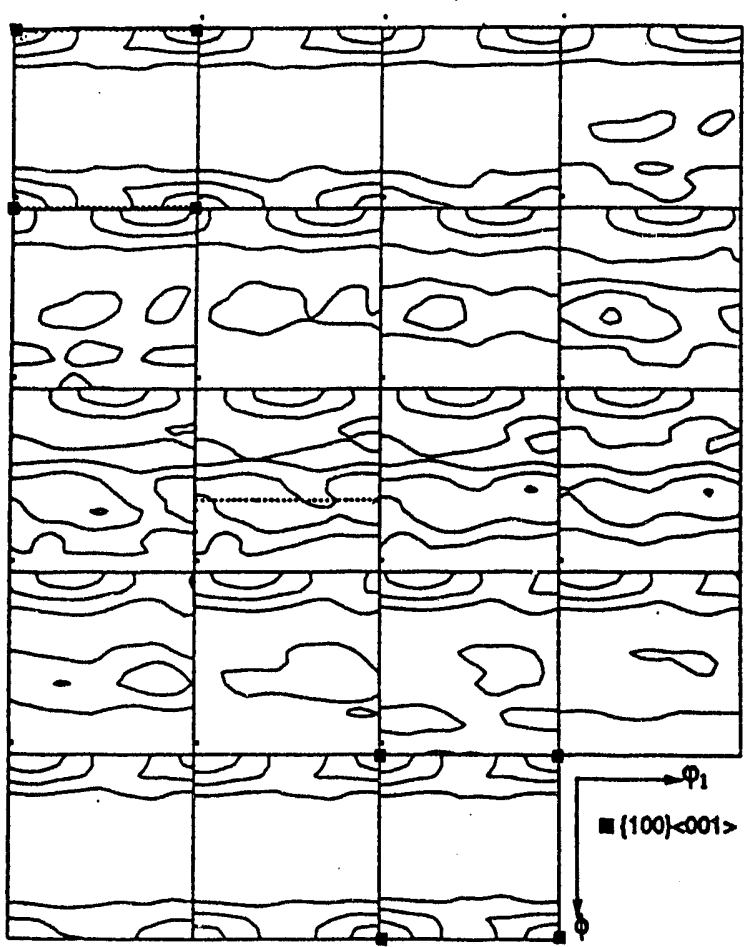

Levels $1,2,3, \ldots . . \quad \varphi_{2}$ sections $0,5, \ldots . .90^{\circ}$

(a)

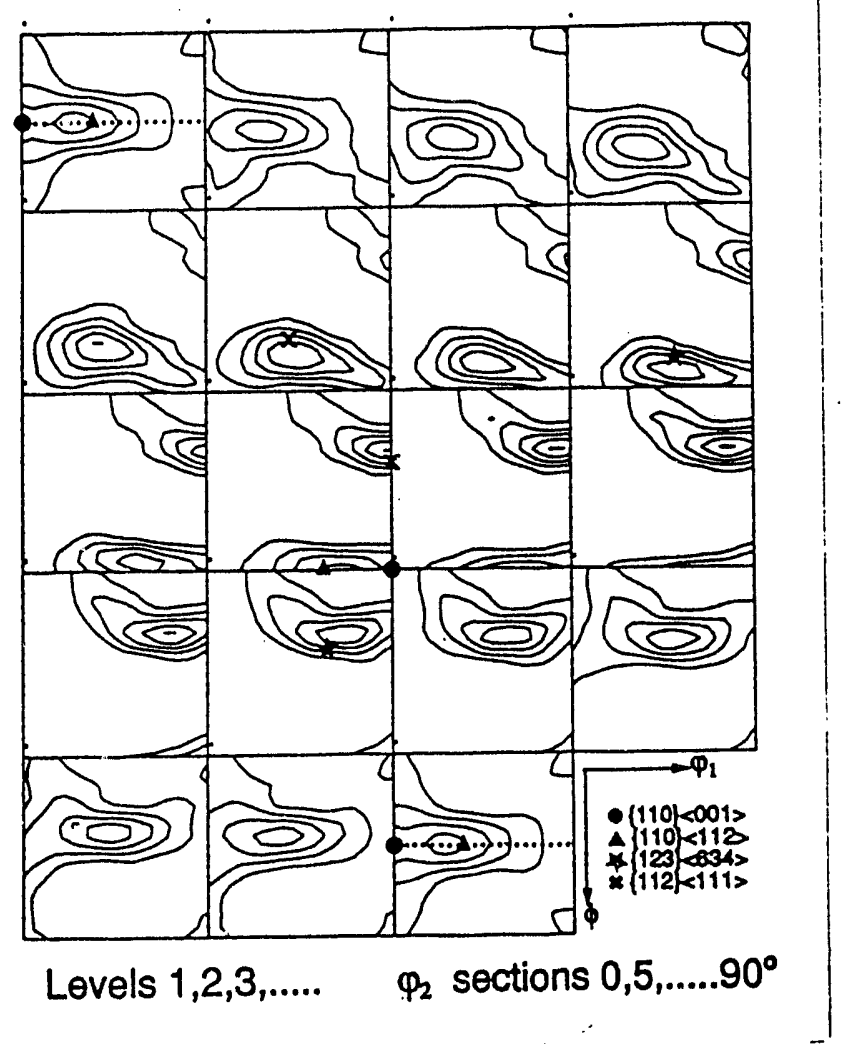

(c)

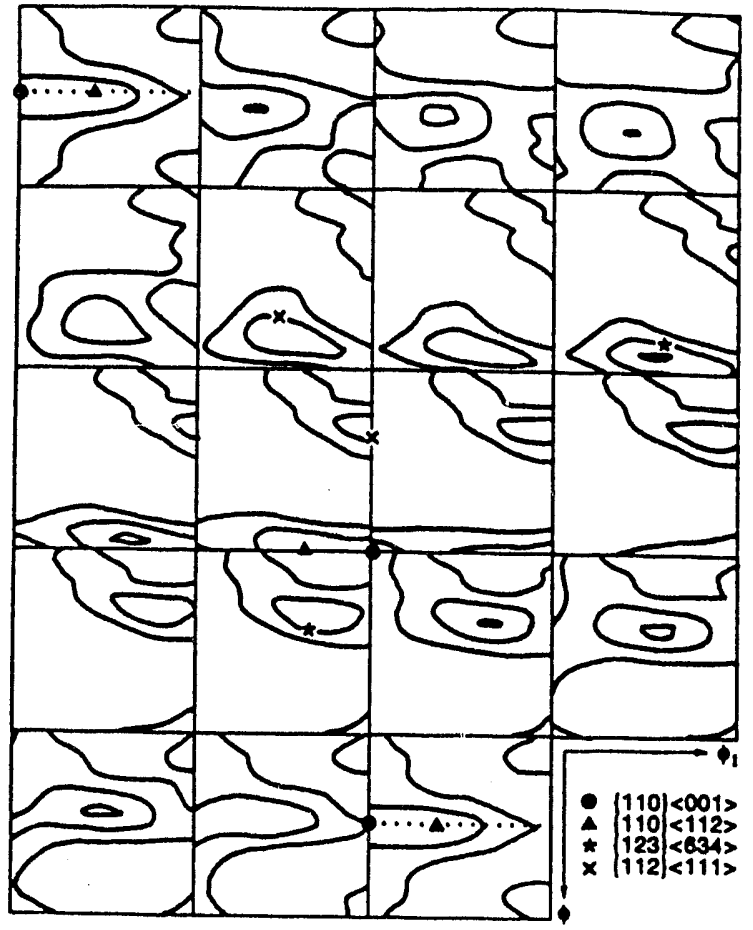

Levels $1,2,3, \ldots . .$. .

(b)

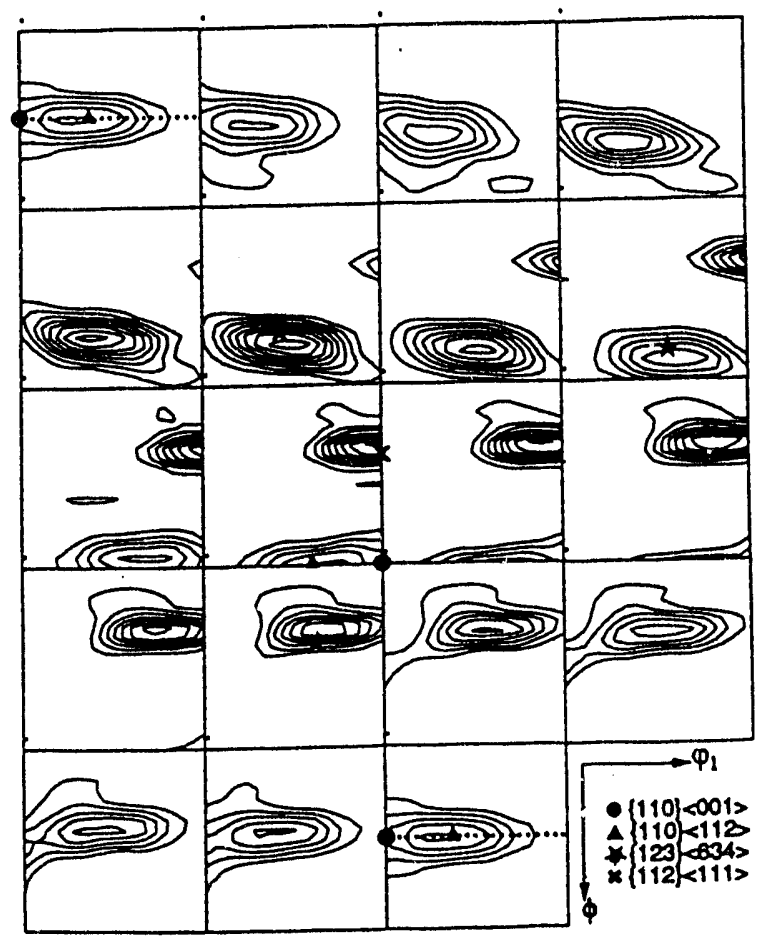

Levels $1,2,3, \ldots . . \quad \varphi_{2}$ sections $0,5, \ldots . .90^{\circ}$

(d)

Fig. 1. Orientation distribution function of nickel following rolling deformation. a) Initial recrystallized texture. b) $37 \%$ reduction, c) $50 \%$ reduction, d) $90 \%$ reduction, e) $98 \%$ reduction (see next page). 


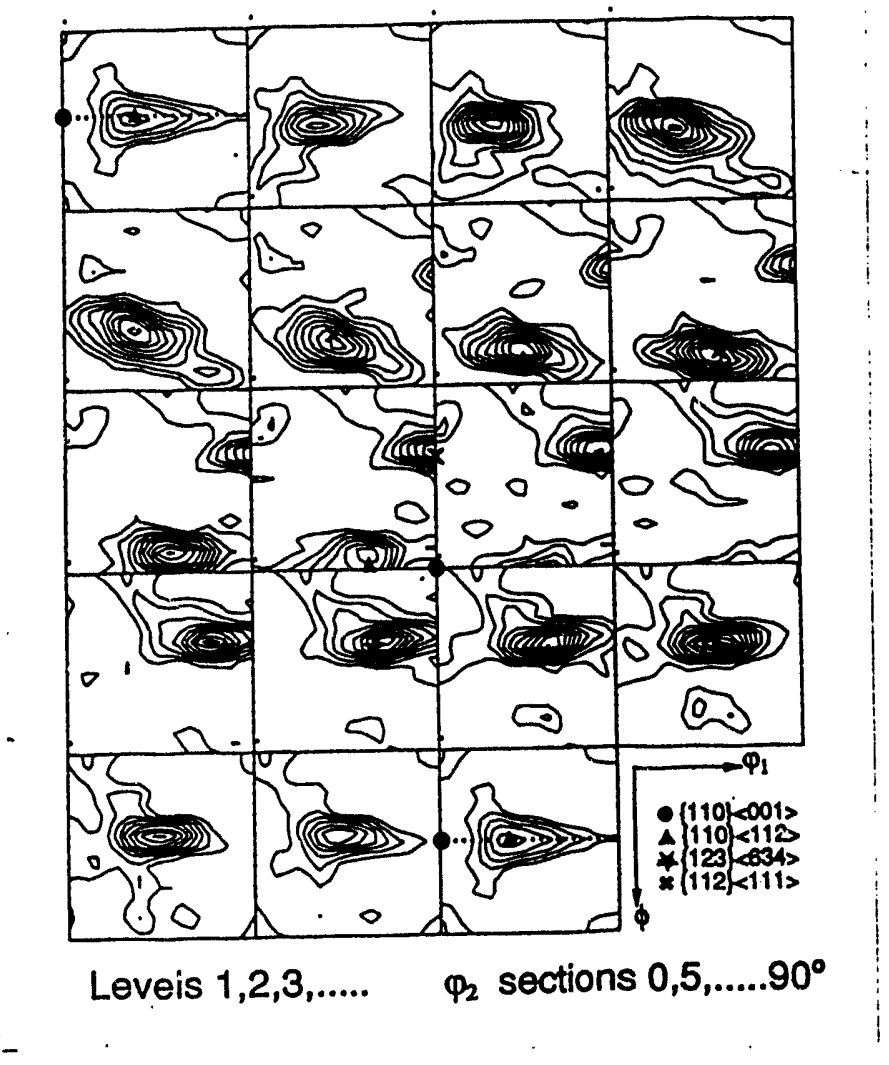

(e)

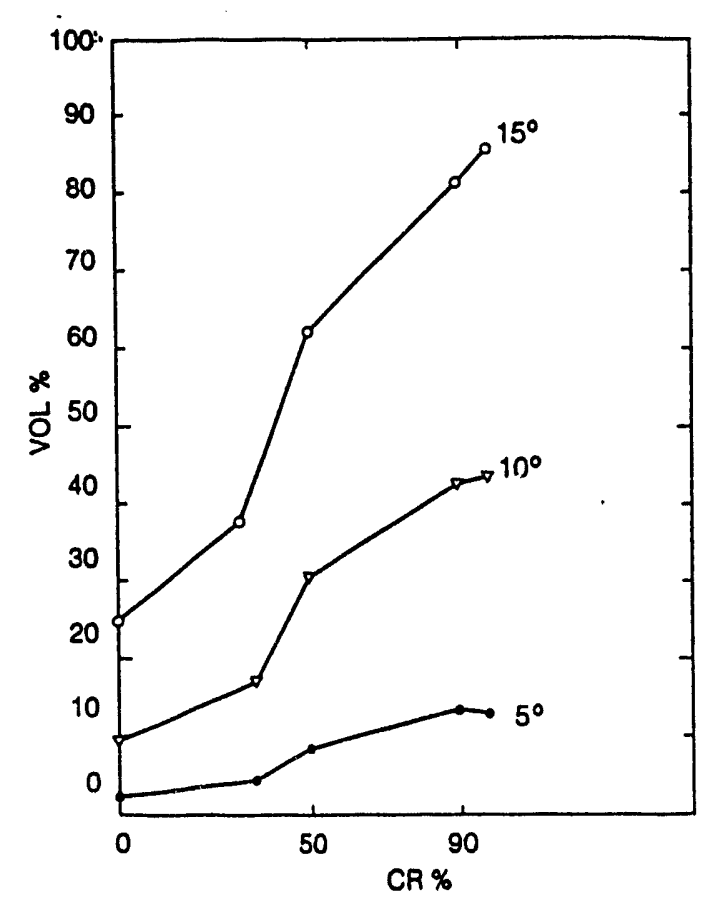

Fig. 2. The sum of the volume concentrations of the three main rolling components ( $\mathrm{S}, \mathrm{C}$ and $\mathrm{B})$. The curves represent the integrated density within $5^{\circ}, 10^{\circ}$ and $15^{\circ}$, respectively, from the rolling components.

cylindrical specimens having a volume of one $\mathrm{cm}^{3}$. For each sample three complete pole figures have been measured and the three dimensional crystallite orientation distribution function (ODF) has been calculated using the series expansion method. The microstructure of the samples has been examined by transmission electron microscopy. Samples have been made from the rolled samples using standard TEM preparation techniques. These sample procedures have also been used to prepare samples from the extremely narrow edge on strips, $0.26 \mathrm{~mm}$ wide, which have been cut from the $98 \%$ rolled sample. Deformation microstructures have been observed at $200 \mathrm{KeV}$ using a JEOL 2000 FX STEM. Each TEM sample has a very large thin area covering from 10 to 20 grains (7).

\section{TEXTURE EVOLUTION}

\subsection{Observations}

Fig. 1 presents the orientation distribution functions for the recrystallized, 37\%, 50\%, $90 \%$ and $98 \%$ cold rolled samples. These quantitative orientation distribution functions are plotted using standard Euler angles $\left(\varphi_{1}, \Phi, \varphi_{2}\right)$. The contour levels are multiples of the normalized random intensity. The initial recrystallized structure has a weak cube texture $\{100\}<001\rangle$ and very weak $\langle 100\rangle$ and $\langle 111\rangle$ fibre components (represented by the dotted lines in Fig. 1a). Following a 37\% cold reduction the texture is still relatively weak but the typical rolling texture has started to form, see Fig. 1b. With increased rolling reductions from $50-98 \%$ the texture develops into a typical rolling texture. The 
$S\{123\}<634>$ component is the dominant component at roughly twice the proportion of the copper $\{112\}<11 \mathrm{~T}>$ component (see Figs. 1c - e). There are similar propertions of copper $\{112\}<11 T>$ and brass $\{110\}<T 12>$ components. Fig. 1 also shows that the actual texture peaks deviate from the ideal texture components by about 10 degs. following $50 \%$ to $90 \%$ reductions. The peak near the copper $\{112\}<<11 \mathrm{~T}>$ texture is spread between the copper $\{112\}<11 \mathrm{~T}\rangle$ and the T component $\{4411\}<11118\rangle$. The peak near the brass $\{110\}<T 12\rangle$ components is shifted to $\{110\}<T 13>$ and $S$ is also shifted away from the ideal. Following $98 \%$ c.r. the peaks have moved closer to their ideal orientations.

The sum of the volume concentrations of the three main texture components ( $\mathrm{S}, \mathrm{C}$ and $\mathrm{B}$ ) is plotted in Fig. 2 as a function of the rolling reduction. Three curves are shown representing the integrated density within $5^{\circ}, 10^{\circ}$ and $15^{\circ}$, respectively, from the orientations of the rolling components. Fig. 2 shows that an increase in strain from 90 to $98 \%$ reduction results in no change in the volume concentration observed within $5^{\circ}$ and $10^{\circ}$ from the three main components. However, a continuous increase in the volume concentration up to the maximum reduction of $98 \%$ is observed for the curve integrated over $15^{\circ}$.

\section{MICROSTRUCTURAL EVOLUTION}

\subsection{Framework}

The evolution of deformation microstructures in high purity nickel during cold-rolling has been reported in recent papers $(5,7)$ where it has been shown that nickel follows a general framework for microstructural evolution suggested for medium and high stacking fault FCC metals (6). Within this framework deforming grains are subdivided into rotated volume elements for all deformation modes. This grain subdivision has been discussed extensively (6) applying the two principles: (i) There are differences in the number and selection of simultaneously acting slip systems among neighbouring volume elements of individual grains. In any one volume element (called a cell block) the number of slip systems falls short of that required for homogeneous (Taylor) deformation but groups of neighbouring cell blocks fulfil the Taylor criterion collectively. (ii) The dislocations are trapped into low-energy dislocation structures in which neighbour dislocations mutually screen their stresses.

The microstructural evolution progresses by subdivision of grains into cell blocks bounded by dislocation boundaries. These boundaries accommodate the lattice misorientations which result from glide on different slip system combinations in neighbouring cell blocks. Such boundaries have been termed geometrically necessary boundaries (8). The cell blocks can be further subdivided by ordinary cell boundaries which have been termed incidental dislocation boundaries since they form as a result of the statistical mutual trapping of glide dislocations supplemented by forest dislocations (8). These two types of boundaries are characterized by parameters such as, macroscopic and crystallographic orientations, misorientation, frequency of occurrence and morphology of the dislocation boundaries.

Consistent and gradual changes occur in the deformation structure with increasing strain. Both the spacing of the geometrically necessary bcundaries and the incidental dislocation boundaries refine and the misorientations across the boundaries increase. The characteristic microstructural features also show a gradual change with strain, which will be illustrated in the following.

\subsection{Microstructural observations}

At low to intermediate strains (37 and 50\% reduction) the microstructure contains cell blocks (CB's) 


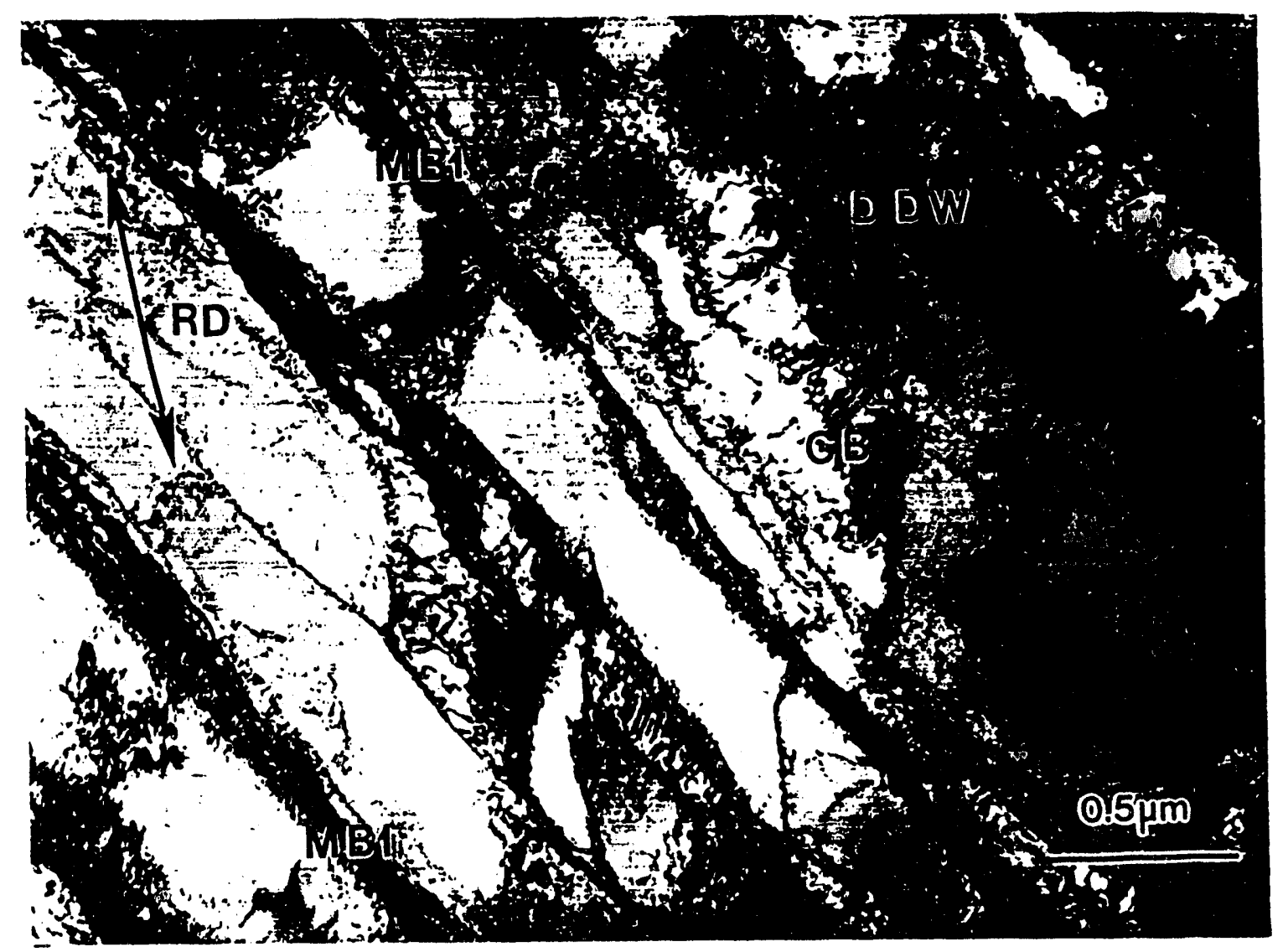

Fig. 3. First generation microbands (MB1s) and dense dislocation walls (DDWs) delineating long cell blocks (CBs) after 50\% reduction. One of the seven CBs is labelled in this photo as well as one of the two DDWs and two of the five MB1s. The rolling direction is marked $\mathrm{RD}$ in this longitudinal side plane view (7). For a difinition of the different structural features, see Ref. 6.

bounded by geometrically necessary boundaries in the form of dense dislocation walls (DDW's) and first generation microbands (MB1s). These features are shown in Fig. 3 for a specimen rolled to $50 \%$ reduction. The CBs at intermediate strains are very elongated with several cells along their length and two to three cells thick. The DDWs and MB1s have a macroscopic orientation with respect to the specimen axes either $30^{\circ}$ or $15^{\circ}$ to the rolling direction. The misorientations across the geometrically necessary boundaries increase with strain from 1 to $3^{\circ}$ observed after $10 \%$ reduction (5) reaching 5 to $10^{\circ}$ after $50 \%$ reduction. In addition to the typical characteristic "low strain" features shown in Fig. 3, dislocation configurations have also been observed which indicate that localized glide has taken place along $\{111\}$ planes. The most characteristic of these features is the S-bands which are composed of a string of S-shaped perturbations in the existing MB1 structure (7).

At large strain ( 70 to $98 \%$ reductions) the structures described above coexist with "large strain" structures over a wide strain range. The most typical large strain structure consists of lamellar bands (lamellae) which are CB's bounded by single boundaries. These lamellar boundaries are geometrically necessary boundaries forming angles of within plus and minus $15^{\circ}$ to the rolling plane. 
The lamellar structure is interspersed with MB1s and equiaxed subgrains.

The fraction of $\mathrm{MB} 1 \mathrm{~s}$ diminishes with increasing strain and at $98 \%$ reduction, no $\mathrm{MB} 1 \mathrm{~s}$ are observed (7). A typical structure following 98\% reduction is shown in Fig. 4 where it is noted that fine lamellar bands alternate with strips of equiaxed subgrains and more widely spaced lamellae. There is an approximate periodicity to the alternating strips with each strip being about 2 - $3 \mu \mathrm{m}$ wide. The periodicity is of the order of one to three grain widths at $98 \%$ reduction.

Misorientations of 5 to $30 \mathrm{deg}$. have been found across several lamellar boundaries. These misorientations have been measured in adjacent lamellae across two grains. The measurements have been made in the longitudinal section along a line parallel to the normal direction of rolling. These misorientations caused a single grain to contain more than one rolling component. For example three different rolling orientations (S, C and Goss) have been observed in the space of $1.5 \mu \mathrm{m}$. The rotation axis for the measured misorientations is frequently close to [T11].

\section{DISCUSSION}

The texture evolution during cold-rolling of pure polycrystalline nickel is in good agreement with previous observations of rolling textures of medium and high stacking fault energy FCC metals (9). This texture evolution has been simulated using different models and relatively good agreement has been found between simulated and experimental textures (9). Such modelling is also part of our ongoing research and we are at present applying the Los Alamos polycrystal plasticity (LApp) code (10). The detailed results of this modelling work will be reported in a forthcoming paper but a preliminary conclusion is that the simulations agree quite well with the present experiments. This agreement is obtained by including the initial texture and by applying Taylor full constraint conditions (FC) at medium strains and relaxed constraint conditions (RC) at large strains $(1,9)$. This change in conditions predicts for example the observed shift from the T-component $\{44$ $11\}\langle 1111 \overline{8}\rangle$ to the C-component $\{112\}<11 T\rangle$ with increasing strain. The observed general agreement between the experimental textures and those predicted according to the Taylor models ( $F C$ and $\mathrm{RC}$ ) shows that the heterogeneous deformation pattern being responsible for the observed grain subdivision may not deviate significantly from the homogeneous deformation suggested by Taylor (11). This may, however, not be surprising due to the suggested governing principles for grain subdivision (see section 3.1), that the number of slip systems in each cell block falls short of the five systems required for homogeneous Taylor deformation, but groups of neighbouring cell blocks fulfil the Taylor criterion collectively. This deformation pattern may, however, fully or partly be the cause of some of the discrepancies seen between predictions and experiments, namely that simulated textures are usually sharper, they may develop with a faster rate, they may show a different density distribution of the major texture components and they may also differ for the minor components $(12,13)$. In the following, only texture sharpness will be considered.

The general finding that simulated textures are normally sharper than experimental textures can be related to different types of inhomogeneous deformation for example caused by: (i) Changes in external geometry during deformation (14), (ii) Different strains in identically oriented grains due to different grain surroundings (9) and (iii) Differences in slip system combinations between different cell blocks within individual grains. The last cause has been shown clearly in the present study by the formation of geometrically necessary boundaries. Such boundaries have been observed as dense dislocation walls and microbands at small to medium strain and as lamellar boundaries at large strain. Thus the grain subdivision will contribute to a scattering of the texture at all strains.

At increasing strain the cell blocks will rotate in accordance with their individual combination of slip systems and the misorientations across the geometrically necessary boundaries will increase. 


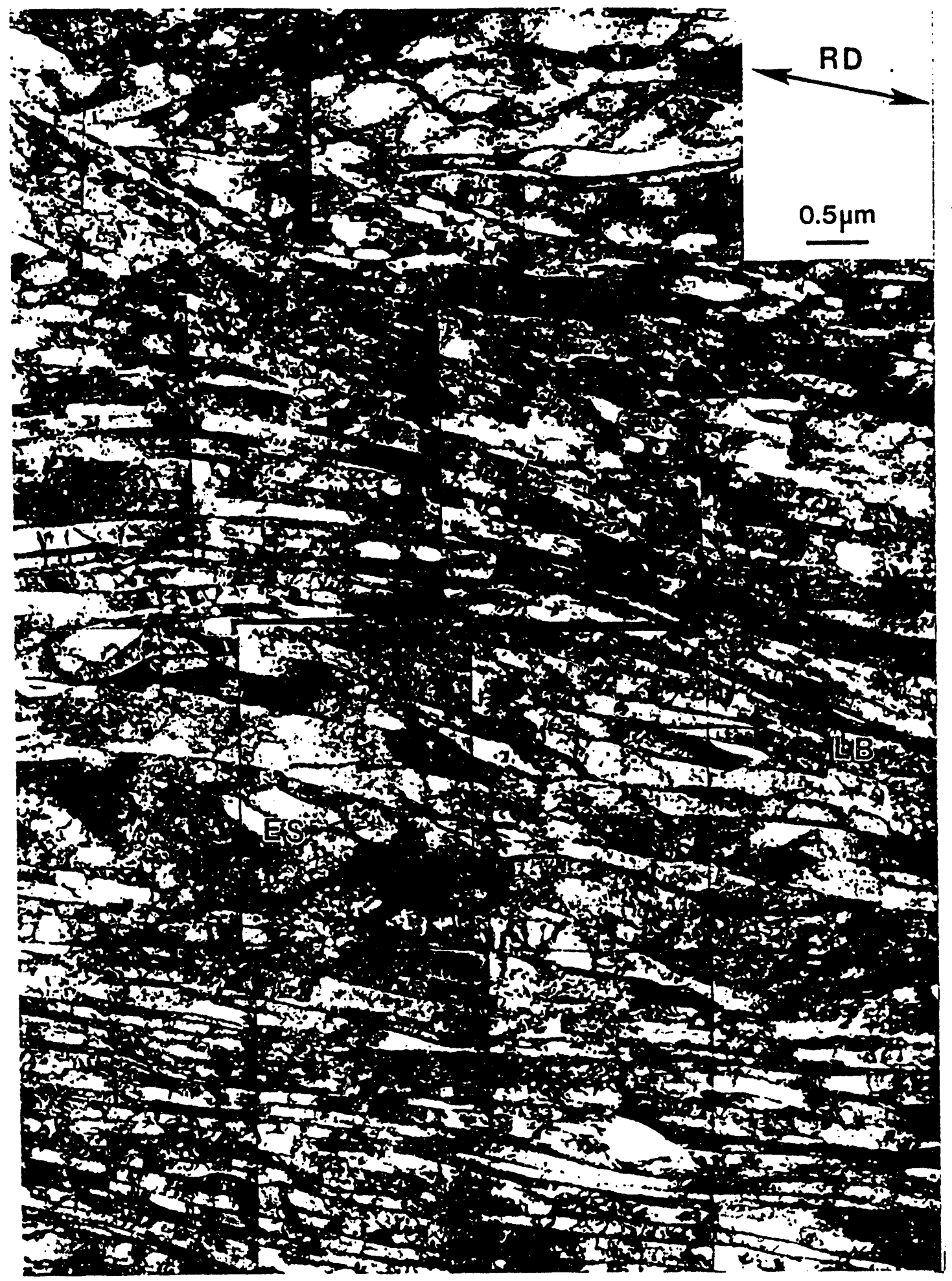

Fig. 4. Large area view across several grains in the longitudinal side plane following $98 \%$ reduction showing a composite microstructure of lamellar boundaries (LB) surrounding strips of equiaxed subgrains (ES). The rolling direction is marked (RD) (7).

This has been observed. As a consequence texture scattering due to (iii) must be expected to increase with strain until the cell blocks have reached stable end orientations. The texture scattering at large strain has been demonstrated by measuring the crystallographic orientations of individual cell blocks. These measurements (although they are quite limited) show that the orientation of many 
component. These measurements also show, in accordance with the observed deformation pattern, that many different texture components are formed by large strain deformation.

Based on the microstructural observations it is suggested that the deformation pattern leading to grain subdivision is incorporated into existing texture models to improve their predictive capability. Research in this direction is being initiated considering an observed grain subdivision on a large scale (14). Such research should be extended also to cover subdivision on a significantly smaller scale (15) in order to simulate the microstructural evolution as observed in the present and in previous work (6).

\section{CONCLUSIONS}

A deformation pattern consisting of grain subdivision on a smaller and smaller scale has been verified microscopically for pure polycrystalline nickel cold-rolled up to $98 \%$ reduction. This deformation pattern affects the texture development.

Texture modelling has been carried out using the LApp code and preliminary results indicate qualitative agreement between preciicted and observed textures. Future texture modelling should incorporate the observed grain subdivision in order to bring model assumptions in accord with the observed microstructural evolution.

\section{ACKNOWLEDGEMENTS}

The authors thank D. Kuhlmann-Wilsdorf and T. Leffers for discussions and E. Sørensen for preparing the manuscript. H. Nilsson, P.B. Olesen and T. Skov for preparing the figures. D. Hughes also acknowledges the support by the United States Department of Energy, DOE, under contract number DE- AC 04 - 76 DP 000789.

\section{REFERENCES}

1) Aernoudt, E., Van Houtte, P. and Leffers, T.: Materials Science and Technology, Ed. H. Mughrabi, CH, Weinheim, 1993, 89.

2) Modelling of Plastic Deformation and Its Engineering Applications, Eds. S.I. Andersen et al., Ris $\varnothing$ National Laboratory, Roskilde, Denmark, 1992, 545 pp.

3) Kuhlmann-Wilsdorf, D.: Mat. Sci. Eng., 1989, A113, 1.

4) Hansen, N.: Mater. Sci. Techn., 1990, 6, 1039.

5) Hughes, D.A. and Hansen, N.: Mater. Sci. Techn., 1991, 7, 544.

6) Hansen, N., Bay, B.B., Hughes, D.A. and Kuhlmann-Wilsdorf, D.: Acta Metall. Mater., 1992, 40, 205.

7) Hughes, D.A. and Hansen, N.: Metall. Trans. A, 1993, 24A, 2021.

8) Kuhlmann-Wilsdorf, D. and Hansen, N.: Scripta Metall. Mater. 1991, 25, 1557.

9) Hirsch, J. and Lücke, K.: Acta Metall., 1988, 36, 2883.

10) Los Alamos Yolycrystal Plasticity Simulation Code: Los Alamos Nat. Lab., LA-CC-88-6, 1988.

11) Taylor, G.I.: J. Inst. Met., 1938, 62, 307.

12) Gil Sevillano, Van Houtte, P. and Aernoudt, E.: Progr. Mat. Sci., 1980, $25,69$.

13) Van Houtte, P.: ICOTOM 6, Ed. S. Nagashima, The Iron and Steel Institute of Japan, Tokio, 1981, 428.

14) Lee, C.S. and Duggan, B.J.: Scripta Metall. Mater., 1993, 28, 121.

15) Leffers, T.: These Proceedings. 


\section{DISCLAIMER}

This report was prepared as an account of work sponsored by an agency of the United States Government. Neither the United States Government nor any agency thereof, nor any of their employees, makes any warranty, express or implied, or assumes any legal liability or responsibility for the accuracy, completeness, or usefulness of any information, apparatus, product, or process disclosed, or represents that its use would not infringe privately owned rights. Reference herein to any specific commercial product, process, or service by trade name, trademark, manufacturer, or otherwise does not necessarily constitute or imply its endorsement, recommendation, or favoring by the United States Government or any agency thereof. The views and opinions of authors expressed herein do not necessarily state or reflect those of the United States Government or any agency thereof. 


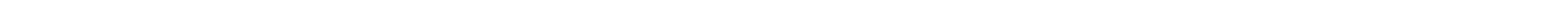


